\title{
Poly(ethylene oxide)-Sodium Dodecyl Sulfate Interactions Studied Using Static and Dynamic Light Scattering
}

\author{
Wyn Brown* and Johan Fundin \\ Department of Physical Chemistry, University of Uppsala, Box 532, \\ 75121 Uppsala, Sweden
}

\section{Maria da Graça Miguel}

Department of Chemistry, University of Coimbra, Coimbra Codex, P-3049 Portugal

Received May 13, 1992; Revised Manuscript Received September 17, 1992

\begin{abstract}
Static and dynamic light scattering measurements have been made on poly(ethylene oxide) (PEO) in the presence of the ionic surfactant sodium dodecyl sulfate (SDS) in aqueous solution over a range of relative concentrations. The purpose was, in part, to demonstrate the polyelectrolyte effects which arise in PEO on formation of the charged complex between the components in solution in which small SDS micelles are incorporated into the flexible PEO coil. The influence of the ionic strength of the medium was studied. Static light scattering measurements show that the radius of gyration changes as a function of SDS concentration and the ionic strength of the medium. Diffusion coefficients from dynamic light scattering (DLS) reflect changes in the pair interaction potential as the SDS and ionic strength are altered. Laplace inversion of the DLS time correlation function gives relaxation time distributions consisting of the PEO/SDS complex as the main component and free SDS micelles as the minor component. Changes in peak areas are related to the increase in the degree of binding between SDS and PEO as the relative concentrations change and also the increase in SDS micellar size with increase in the ionic strength of the medium.
\end{abstract}

\section{Introduction}

The charged complex formed between the ionic micelleforming surfactant sodium dodecyl sulfate (SDS) and the neutral polymer poly(ethylene oxide) (PEO) has received much attention in the literature. Similar complexes are formed between surfactants and other polymers, for example, hydroxypropyl cellulose, ${ }^{5}$ poly(vinylpyrrolidone), ${ }^{6}$ and ethyl hydroxyethyl cellulose ${ }^{7}$ with the interaction between the two species apparently being more favored the greater the hydrophobicity of the polymer. Recent reviews of the interactions between nonionic polymers and ionic surfactants have been given by Goddard, ${ }^{1}$ Saito, ${ }^{2}$ Hayakawa and Kwak, ${ }^{3}$ and Thalberg and Lindman. ${ }^{4}$

In the PEO/SDS system a number of different techniques have been employed to study the nature of the charged complex formed (see, for example, the pioneering work of Jones ${ }^{8}$ on surface tension, electrical conductivity, and viscosity). In solutions of $P E O$ containing sufficiently low concentrations of SDS such that a critical level is not exceeded, most of the surfactant molecules apparently exist freely in the solution. When the SDS concentration exceeds this level, termed the critical aggregation concentration (cac), ${ }^{9}$ complex formation occurs over a broad SDS range in which a substantial amount of SDS is bound in the form of micelles to the polymer chain, although a proportion of SDS will still be present in the free form. As discussed by Cabane, ${ }^{10}$ the amount of SDS bound to PEO remains in constant proportion to the PEO concentration. The SANS measurements of Cabane and coworkers ${ }^{10}$ show that the micelles which form on the PEO chains are probably small (of the magnitude $20 \AA$ in radius) and the polymer is associated with the interface between the hydrocarbon and water, i.e. the polymer is sited close to the surface of the SDS aggregates and the chain segments mainly interact in the surfactant head group region; this is tantamount to solubilization of segments of the flexible PEO chain in the SDS micelle. The critical micelle concentration $(\mathrm{cmc})$ is approximately $8 \mathrm{mM}\left(0.23 \times 10^{-2}\right.$ $\mathrm{g} \mathrm{mL}^{-1}$ ) for SDS in the salt-free and polymer-free system and decreases strongly with increasing ionic strength. In the presence of $\mathrm{PEO}$, the cac values are substantially lower than the cmc of pure SDS. Above the concentration at which the PEO has become saturated with SDS micelles, the concentration of free micelles increases in the bulk solution as indicated by the phase diagram given by Cabane. ${ }^{10}$ Fluorescence decay measurements have been used to establish the micellar aggregation number in the complex, e.g. refs 11 and 12 . At the cac the aggregation numbers are low: only about $1 / 3$ of the aggregation number of free SDS micelles in the polymer-free solution. The average aggregation number in the complex then increases on further addition of SDS to about 60 at saturation, ${ }^{12}$ the clusters growing both in size and number. At saturation, there is a distance of approximately $90 \AA$ between adjacent micelles in salt-free solution and about $60 \AA$ at $0.8 \mathrm{M}$ salt. ${ }^{10}$

Increasing the ionic strength of the solution leads to a larger size of the bound micelles although these are still smaller than free micelles at the same ionic strength. The molecular weight of the polymer has little influence on the interaction as long as it exceeds a minimum value of about 4000 . However, there are only speculative interpretations of the mechanism of interaction.

The hydrodynamic behavior of PEO-SDS complexes (employing viscosity, conductometry, ultracentrifugation methods) has been investigated by Francois et al. ${ }^{13}$ These workers showed that the saturated complex has properties which resemble those of a polyelectrolyte of similar charge density. They estimated, using ultracentrifugation, that the molecular weight of the complex at saturation is about 3.5 times greater than that of the original polymer in approximate agreement with the earlier dialysis results of Shirahama. ${ }^{14}$ These figures are in agreement with the expectation from the phase diagram given in ref $10 \mathrm{~b}$.

Light scattering techniques are highly sensitive and noninvasive methods for investigating changes in the conformation of the complex but, surprisingly, have been little employed in this context. Light scattering investigations having close relevance to the present study, since they reveal interesting properties of polymer-SDS inter- 
actions, are those of the coil-globule transition in solutions of the polymer poly ( $N$-isopropylacrylamide) with SDS by Meewes et al. ${ }^{16}$ and Ricka et al. ${ }^{17}$ Communications from the group of Dubin ${ }^{18}$ have described the properties of polyelectrolyte/mixed micellar complexes. A recent contribution of Dubin et al. ${ }^{15}$ examined the influence of the micellar counterion on the interaction of PEO with dodecyl sulfate micelles, this study being stimulated by the observation that the cmc-lowering effect of the polymer is strongly dependent on the nature of the cation. The present communication utilizes light scattering techniques (a) to demonstrate the formation of the PEO-SDS charged complex whereby polyelectrolyte properties are imparted to the neutral PEO chain, (b) to elucidate the composition of the charged complex in the presence of a simple salt, and (c) to investigate the modification of the polyelectrolyte properties of the complex when excess SDS is present. Static light scattering is thus used to estimate the radius of gyration. Dynamic light scattering is used to obtain the hydrodynamic radius at infinite dilution and the relaxation time distribution under a variety of conditions. A monodisperse PEO sample with $\mathrm{MW} \simeq 10^{6}$ has been employed. Although it is known that the polymer MW has little or no influence on the aggregation number of SDS, it is convenient to use a large MW in order to gain precision in determination of radii of gyration, etc.

\section{Experimental Section}

Materials. Poly(ethylene oxide) (PEO) designated SE-150 $\left(M_{\mathrm{w}}=9.96 \times 10^{5} ; M_{\mathrm{w}} / M_{\mathrm{n}}=1.12\right.$ from Toya Soda, Ltd., was used. SDS was from BDH, U.K., and had a cmc in agreement with literature values $(8 \mathrm{mM})$ and was used without further purification. Aqueous solutions were filtered through $0.22 \mu \mathrm{m}$ Millipore (Fluoropore) "Millex" filters directly into the $15 \mathrm{~mm}$ diameter cylindrical light scattering cells, which were then sealed.

Dynamic Light Scattering (DLS). DLS measurements have been made using the apparatus and techniques described in an earlier communication. ${ }^{19}$ The data were assembled using an $A L V$ wide-band, multi-tau, digital autocorrelator with 23 simultaneous sampling times allowing characterization of relaxation time distributions extending over 9 decades in time.

Data Analysis. In the analysis of the measured autocorrelation curves, an inverse Laplace transformation (ILT analysis) was performed employing the algorithm REPES $^{20}$ to obtain the distribution of relaxation times. This program is similar to Provencher's CONTIN ${ }^{21}$ except that the former directly minimizes the sum of the squared differences between experimental and calculated intensity-intensity $g_{2}(t)$ correlation functions using nonlinear programming. For a system exhibiting a distribution of relaxation times, the field correlation function $g_{1}(t)\left(g_{2}(t)=\right.$ $\left.g_{1}^{2}(t)+1\right)$ is described by a continuous function of the relaxation time $\tau$ using the Laplace transform

$$
g_{1}(t)=\int_{0}^{\infty} A(\tau) e^{-t / \tau} \mathrm{d}(\tau)
$$

Relaxation time distributions are given, for example in Figure 11 , in the form of $\tau A(\tau)$ versus $\log \tau$ plots, with $\tau A(\tau)$ in arbitrary units. This provides an equal area representation. ${ }^{22}$

The range of relaxation times allowed in the fitting was usually between $0.5 \mu \mathrm{s}$ and $1 \mathrm{~s}$ with a density of 12 points per decade. Relaxation rates are obtained from the moments of the peaks in the relaxation time distribution or, if the peaks overlap, from the peak maximum position. With a broad distribution of relaxation times, these inversion methods yield multiple peaks in the "unsmoothed" analysis. The "smoothing" parameter $(P)$ was selected as 0.5 in all cases, after it was established that the number of peaks did not change with further increase in smoothing. As a further check, an analysis was made on a simulated correlation function consisting of a broad continuous distribution of relaxation times with noise added equal to the residuals from the analysis of the experimental correlation curve. REPES recovers

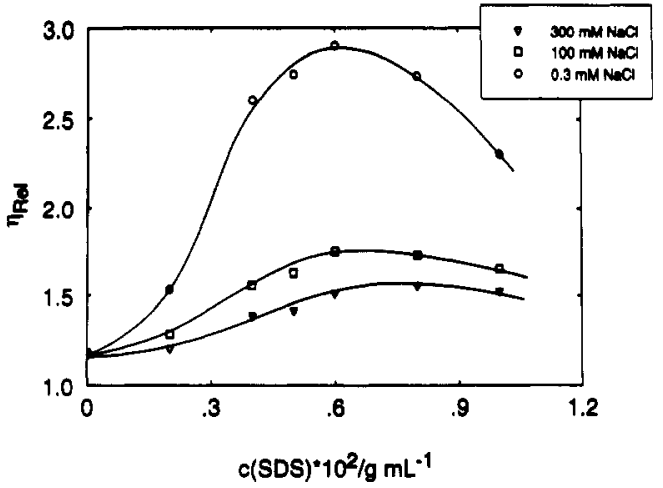

Figure 1. Diagram of the relative viscosity $\left(25^{\circ} \mathrm{C}\right)$ versus concentration SDS, at a constant concentration $C_{\mathrm{PEO}}=0.1 \%$ at different ionic strengths as shown. The data show a strong polyelectrolyte effect with a peak at about $C_{\mathrm{SDS}} / C_{\mathrm{PEO}} \approx 6$.

the original distribution except when a very low smoothing parameter $(P \approx 0)$ is used. ${ }^{21}$ Diffusion coefficients were calculated as $\left(D=\Gamma / q^{2}\right)_{q \rightarrow 0}$, where $q$ is the scattering vector and the relative amplitudes are obtained from the moments of the peaks and are given in the output of the program. $\Gamma$ is the measured relaxation rate $\left(\Gamma=\left(\tau^{-1}\right)\right)$.

Static Light Scattering. Static light scattering measurements were performed with a photon-counting device supplied by Hamamatsu. The light source was a $3-\mathrm{mW} \mathrm{He}-\mathrm{Ne}$ laser $(632$ $\mathrm{nm}$ ). The reduced scattering intensity $K C / R_{\theta}$ was determined for the same solutions as used for dynamic light scattering measurements. It should be noted that $C$ in $K C / R_{\theta}$ and $(\mathrm{d} n / \mathrm{d} C)$ refer in this work to the PEO component, since the structure of the complex between PEO and SDS is unknown. The value of $(\mathrm{d} n / \mathrm{d} C)=0.134 \mathrm{~mL} / \mathrm{g}$ for PEO was used. ${ }^{23}$ Thus concentrations are given in terms of the PEO component for convenience in scaling of the data. Radii of gyration $\left(R_{\mathrm{g}}\right)$ were estimated from the ratio of the slope to intercept of linear plots of the angular dependence of the reduced scattering intensity $\left(K C / R_{\theta}\right)$ at the arbitrary concentration of $C_{\mathrm{PEO}}=0.1 \%(\mathrm{w} / \mathrm{v})$ as shown for typical data in Figure 2. Benzene was used for calibration with the Rayleigh ratio for benzene $=8.51 \times 10^{6} \mathrm{~cm}^{-1}$ (ref 24).

Surface Tension Measurements. Surface tension measurements were made using an instrument based on the dropvolume principle described by Tornberg. ${ }^{25}$

\section{Results and Discussion}

Viscosity. Viscosity measurements on dilute solutions were made using an Ubbelohde capillary viscometer with $\mathrm{NaCl}$ and SDS present in the aqueous solvent. The relative viscosity is used here rather than the more usual reduced viscosity $\left(\eta_{\mathrm{sp}} / C\right)$ since the concentration of the complex is unknown. Relative viscosities were determined at a fixed PEO concentration of $0.1 \%(w / v)$ as a function of $C_{\text {SDS }}$ at three concentrations of simple salt. The data shown in Figure 1 demonstrate the strong coil expansion at the lowest salt concentration which reaches a maximum at $C_{\mathrm{SDS} /} C_{\mathrm{PEO}} \approx 6$. This observation, and those described below, contrast with that of Cabane and Duplessix ${ }^{10 b}$ that there is only a slight expansion of the coil dimensions as the ionic strength is decreased. The maximum is broad on the $C_{\mathrm{SDS}}$ scale, and this is in accord with, for example, Figures 3 and 7.

Static Light Scattering. Static light scattering measurements have been made on solutions of PEO $\left(M_{\mathrm{w}}=\right.$ $\left.9.96 \times 10^{5}\right)$ at a series of salt $(\mathrm{NaCl})$ concentrations in the presence of SDS. Since the polyelectrolyte properties of the polymer-SDS complex depend sensitively on the extent of binding of SDS, it is necessary to make experiments at different values of the ratio $\left(C_{\mathrm{SDS}} / C_{\mathrm{PEO}}\right)$. Solutions were thus prepared in the PEO concentration range $0.02-0.1 \%$ for each $\left(C_{\mathrm{SDS}} / C_{\mathrm{PEO}}\right)$ value and at each salt concentration. 


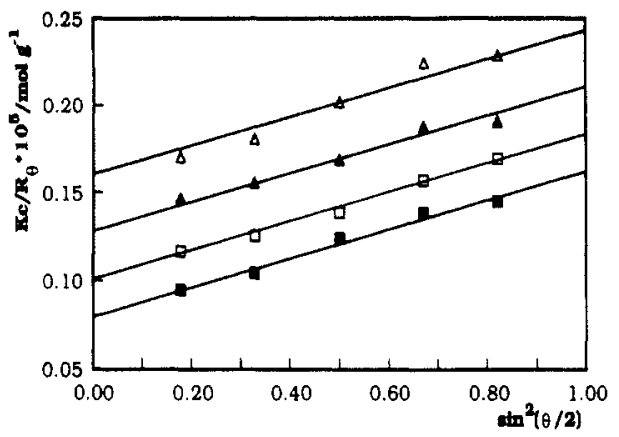

Figure 2. Intensity light scattering. Reduced scattering intensity versus $\sin ^{2} \theta / 2$ with a constant ratio $C_{\mathrm{SDS}} / C_{\mathrm{PEO}}=5$. Concentrations of PEO were as follows: $0.10 \%(\Delta) ; 0.08 \%$ (A); $0.06 \%$ (ㅁ);0.04\% (D). Data were obtained at $25^{\circ} \mathrm{C}$ and at ionic strength $0.05 \mathrm{M}$.

The information obtainable from static LS is limited by the fact that $C_{\text {complex }}$ and $(\mathrm{d} n / \mathrm{d} C)_{\text {complex }}$ are unknown. Thus the molecular weight of the complex is not directly accessible and neither is the corresponding second virial coefficient. The method for treating the light scattering data suggested by Dubin et al..$^{26}$ is not viable for obtaining the molecular weight of the complex in absence of knowledge concerning its composition. Since the present purpose is only to semiquantitatively demonstrate the polyelectrolytic character imparted to the PEO chain on complexing with SDS, the weight concentration, $C$, appearing in $K C / R_{\theta}$ is referred in the present experiments to the PEO component itself. We have arbitrarily selected data at $C_{\mathrm{PEO}}=0.1 \%$ (from each diagram similar to that in Figure 2) to use for the evaluation of $R_{\mathrm{g}}$ at each SDS/ PEO ratio and at each $\mathrm{NaCl}$ concentration; i.e. $\left(R_{g}\right)_{\text {apparent }}$ values were determined from the slopes of the plots of the reduced scattering intensity $\left(K C / R_{\theta}\right)$ versus $\sin ^{2}(\theta / 2)$ for the line corresponding to $C_{\mathrm{PEO}}=0.1 \%$. Strictly speaking, the ratio $\left(C_{\text {bound-SDs }} / C_{\mathrm{PEO}}\right.$ ) should be the quantity maintained constant, but this is difficult to achieve in practice since $C_{\text {bound-SDs }}$ is not known precisely. However, the linearity of the static LS data such as those shown in Figure 2 suggests that the approximation may be justified, at least at higher ionic strengths, a conclusion which is supported by the data of Francois et al. ${ }^{13}$ in the estimation of the intrinsic viscosity of the charged complex for the saturated complex in the plateau region.

Figure 3 shows the resulting $R_{\mathrm{g}}$ data for SDS/PEO ratios 1 to 10 and these clearly demonstrate the polyelectrolytic character of the PEO-SDS complex, with a strong change in the coil expansion as the ionic strength of the medium is lowered. The maximum as a function of $C_{\mathrm{SDS}}$ is weak but lies in the vicinity of $C_{\mathrm{SDS}} / C_{\mathrm{PEO}}=6$.

Figure 4 shows that the overlap concentration $C^{*}(=3 M$ / $4 \pi R_{\mathrm{g}}{ }^{3} N_{\mathrm{A}}$ ) is well exceeded at all SDS concentrations at the lowest salt concentration, but only at the maximum binding degree of $C_{\mathrm{SDS}} / C_{\mathrm{PEO}} \approx 6$ in $0.05 \mathrm{M} \mathrm{NaCl}$. When the SDS concentration exceeds this value, $C / C^{*}<1$ owing to screening effects from free $\mathrm{Na}^{+}$ions.

Dynamic Light Scattering. Diffusion coefficients were determined from the measured decay rate $\Gamma$ as $D=$ $\left(\Gamma / q^{2}\right)_{q \rightarrow 0}$, where $q$ is the scattering vector, after it had first been determined that $\Gamma$ was linearly dependent on the scattering vector $q^{2}$, passing through the origin, demonstrating a diffusive process.

Figure 5 shows typical data for the concentration dependence of $D$ on $C_{\mathrm{PEO}}$, at different values of the ratio of SDS/PEO and at a simple salt concentration of $0.1 \mathrm{M}$. A constant ratio between the SDS and PEO concentration

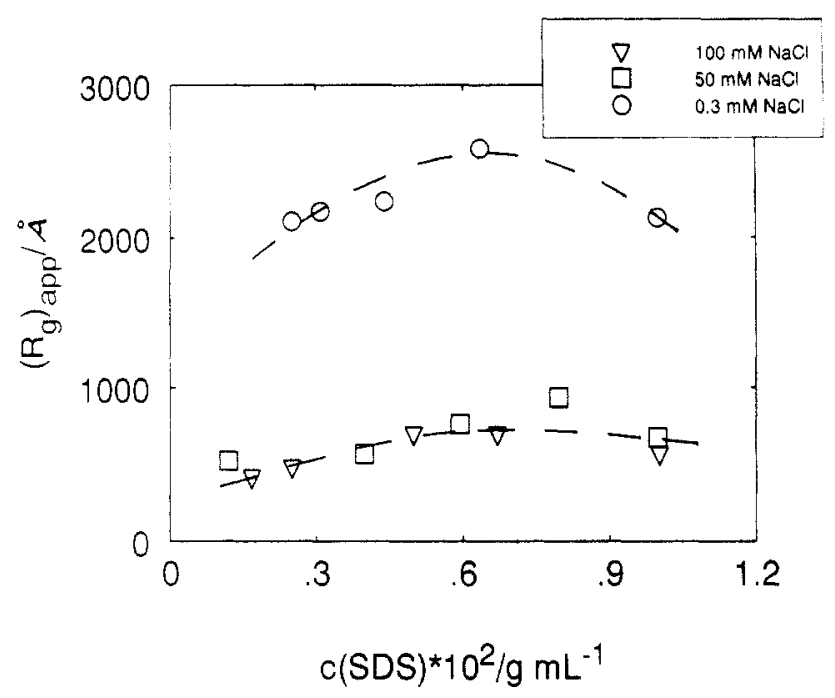

Figure 3. Apparent radius of gyration $\left(R_{z}\right)$ versus $C_{\text {SDS }}$ at a constant concentration $C_{\mathrm{PEO}}=0.1 \%$ and $25^{\circ} \mathrm{C}$ at different ionic strengths. $R_{f}$ is determined at a constant PEO concentration of $0.1 \%$. The dashed lines are guides to the eye.

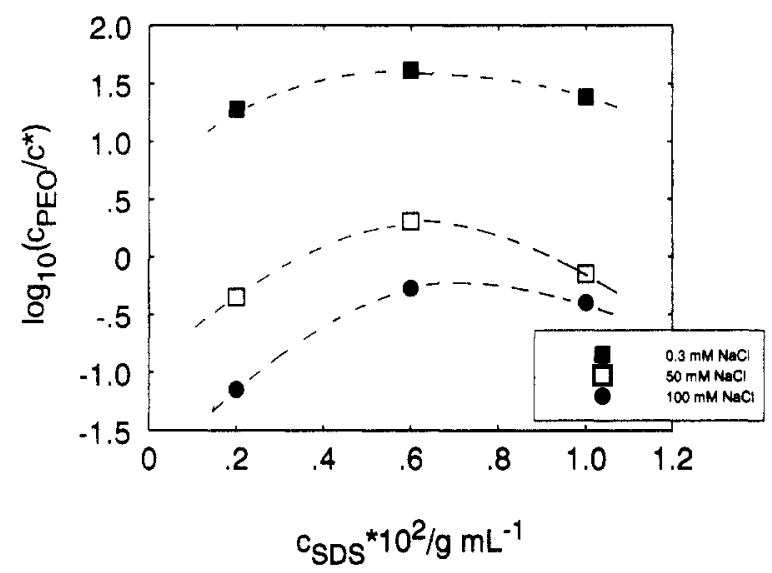

Figure 4. Degree of overlap $\left(C / C^{*}\right)$ for PEO/SDS complex: log $\left(C / C^{*}\right)$ versus $C_{\mathrm{SDS}}$ at different ionic strengths for $C_{\mathrm{PEO}}=0.1 \%$. Dashed lines are guides to the eye.

is used in order to approximate a constant binding degree at each PEO concentration.

The hydrodynamic virial coefficient, $k_{\mathrm{D}}$, is defined as the concentration coefficient in $D=D_{0}\left(1+k_{\mathrm{D}} C+\ldots\right) ; D_{\mathrm{o}}$ is the value of $D$ at infinite dilution

$$
k_{\mathrm{D}}=2 A_{2} M-k_{\mathrm{f}}-2 v_{2}
$$

where $k_{\mathrm{f}}$ describes the concentration dependence of the friction coefficient, $f, f=f_{0}\left(1+k_{\mathrm{f}} C+\ldots\right)$, and $v_{2}$ is the partial specific volume.

$k_{\mathrm{D}}$ is thus the sum of a static factor proportional to the second virial coefficient, $A_{2}$, and the concentration dependence of the friction coefficient. Expressions for $k_{\mathrm{f}}$ have been given and are summarized in ref 27. For a model of interacting hard spheres, the pair interaction potential describing the virial coefficient is usually given by the DLVO theory as the sum of a replusive interaction due to the charges on the spheres and an attractive interaction (van der Waals forces). Approximate expressions are obtained by solution of the Poisson-Boltzmann equation in the limiting cases. Thus $k_{D}$ initially increases strongly as shown in Figure 6a as the repulsive contributions to the interaction potential due to the fired charges become increasingly important. Subsequently there is a decrease due to the screening effect of free $\mathrm{Na}^{+}$counterions from the excess SDS which progressively increase in concentration after the PEO chains are saturated. Figure $6 \mathrm{~b}$ 
$c(P E O) / C(S D S)=1 / 3$

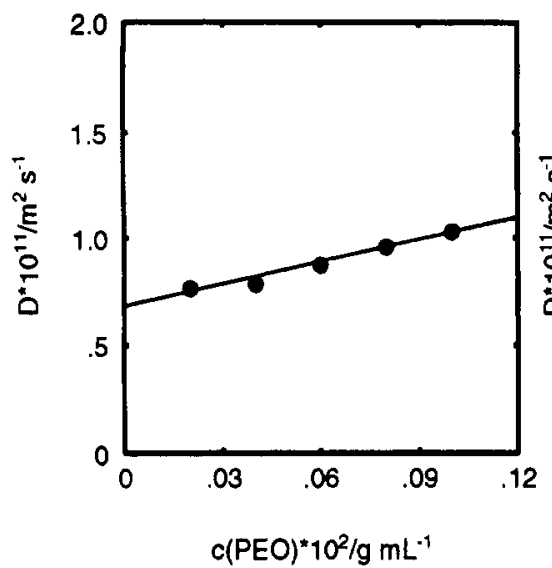

$c(P E O) / C(S D S)=1 / 6$

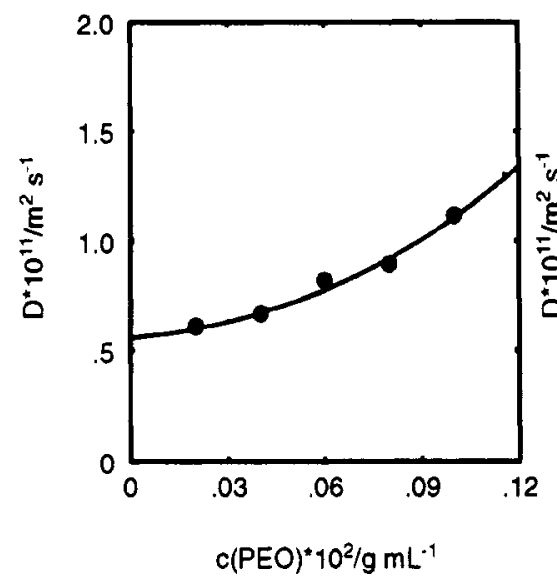

$\mathrm{C}(\mathrm{PEO}) / \mathrm{c}(\mathrm{SDS})=1 / 4$

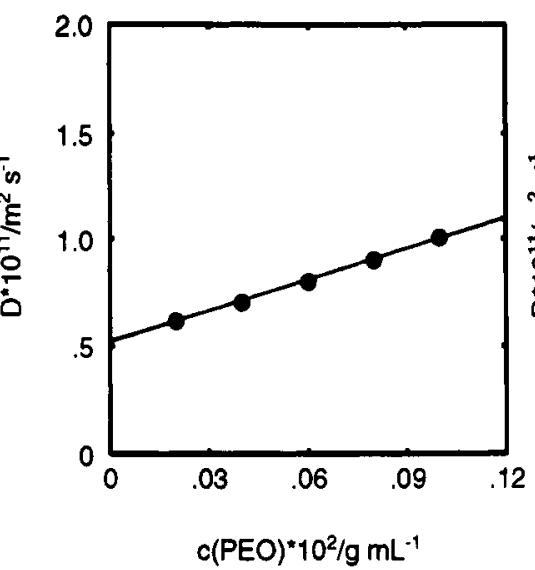

$c($ PEO $) / c($ SDS $)=1 / 7$

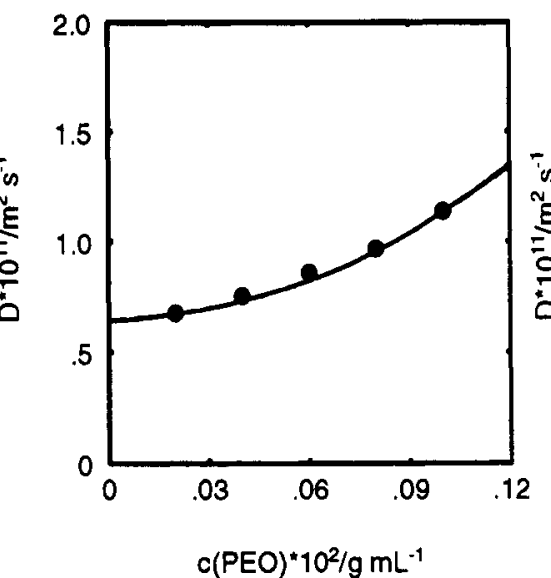

$c($ PEO $) / c(S D S)=1 / 5$

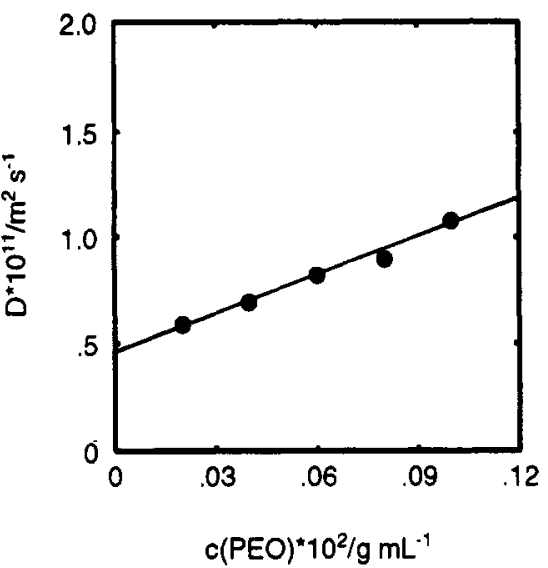

$c($ PEO $) / c(S D S)=1 / 9$

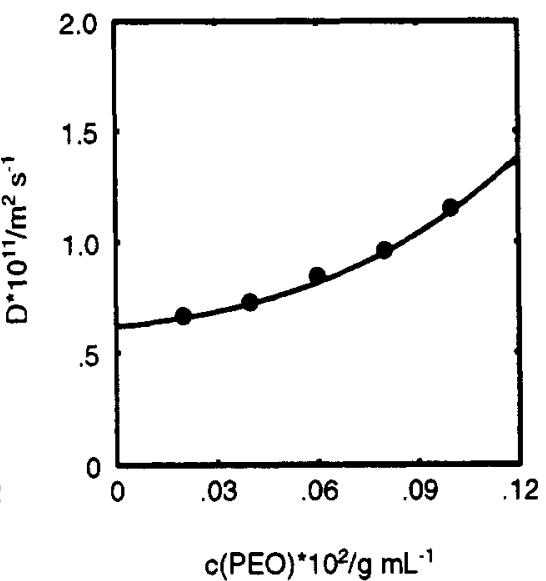

Figure 5. Diffusion coefficients as a function of $C_{\mathrm{PEO}}$ at different values of $C_{\mathrm{SDS}} / C_{\mathrm{PEO}}$ and ionic strength $0.1 \mathrm{M}$. Measurements at $25^{\circ} \mathrm{C}$ and angle $60^{\circ}$.

shows the corresponding variation of the second virial coefficient $A_{2}$ with the ratio $C_{\mathrm{SDS}} / C_{\mathrm{PEO}}$. The trend in $A_{2}$ follows closely that in $k_{\mathrm{D}}$ in Figure 6a. It is noted here in Figure $6 \mathrm{~b}$, that the apparent second virial coefficient is used where the modification "apparent" enters since the $K C / R_{\theta}$ values used in evaluation refer to the PEO component rather than the charged complex. However, the trends in the data should be closely similar.

Hydrodynamic radii for the complex at each $C_{\mathrm{SDS} / \mathrm{PEO}}$ value were obtained at the limit of $C_{\mathrm{PEO}}=0$ from the data in Figure 5 in $0.1 \mathrm{M} \mathrm{NaCl}$ according to the Stokes-Einstein equation: $R_{\mathrm{H}}=k T / 6 \pi \eta_{0} D_{0}$. These are shown in Figure 7 where $R_{\mathrm{H}}$ is plotted versus $C_{\mathrm{SDS}} / C_{\mathrm{PEO}}$. The location of the maximum in the hydrodynamic radius gives more precisely the point at which the binding reaches saturation than does the relative viscosity or intensity light scattering data. These data suggest a maximum at $C_{\mathrm{SDS}} / C_{\mathrm{PEO}}=5$ in $0.1 \mathrm{M} \mathrm{NaCl}$ (i.e. $17.4 \mathrm{mM} \mathrm{SDS}\left(0.5 \times 10^{-2} \mathrm{~g} \mathrm{~mL}^{-1}\right)$ with $0.1 \% \mathrm{PEO}$ ). Saturation at a concentration ratio $C_{\mathrm{SDS}} /$ $C_{\mathrm{PEO}} \approx 5$ is higher than the value suggested by the phase diagram of Cabane and Duplessix. ${ }^{10 \mathrm{~b}}$ However, the latter was made for the salt-free system. It was shown in ref $10 \mathrm{~b}$ that the saturation level increases with increasing ionic strength.

Figure 8a depicts the influence of low molar mass salt on the diffusion coefficient where the data are displayed as a function of $C_{\mathrm{PEO}}$ for SDS/PEO at the saturation value of 5. The figure shows that the interaction potential decreases with ionic strength; i.e. $k_{\mathrm{D}}$ decreases with increasing ionic strength due to screening of the fixed charges by low molecular weight ions, which is most probably the main effect. There will, however, be a more expanded PEO conformation as the ionic strength is increased due to the enhanced binding of SDS, and this effect also contributes to the smaller $D$ values.

Figure $8 \mathrm{~b}$ shows the accumulated data from plots of the type of Figure $8 \mathrm{a}$ for a range of $C_{\mathrm{SDS}} / C_{\mathrm{PEO}}$ values. The overall form of the curves shown in Figure $8 \mathrm{~b}$ is dependent on the screening effect of free ions on the interactions between the fixed charges on the bound micelles; i.e. it is simply related to the pair interaction potential. $D$ initially increases with $C_{\text {SDS }}$ as the degree of binding and hence the interaction potential increase up to the point of saturation. Subsequently, $D$ decreases at a given ionic strength owing to screening by free $\mathrm{Na}^{+}$counterions. These changes are most pronounced at the lowest ionic strength $(0.3 \mathrm{mM})$, where the maximum value of $D$ is attained at $C_{\mathrm{SDS}} / C_{\mathrm{PEO}}$ $\approx 4$. This value agrees approximately with that of ref $10 \mathrm{~b}$ in salt-free solution. Increased screening by the low MW salt leads to a lower $D$ value for a given SDS concentration and the maximum thus moves to higher SDS values as anticipated from the phase diagram in ref $10 \mathrm{~b}$.

Relaration Time Distributions. Relaxation time distributions obtained using inverse Laplace transformation (ILT) with the algorithm REPES (Experimental Section) are shown in Figure 9 for data over a range of $C_{\mathrm{SDS}} / C_{\mathrm{PEO}}$ up to 20 . As shown in this and the subsequent diagrams, peaks are registered for both the PEO complex and free SDS micelles. Thus the lower intensity peak at shorter relaxation time represents free SDS micelles and the main peak the PEO/SDS complex. The estimated hydrodynamic radius as indicated for the SDS peak agrees 



Figure 6. (a, top) Hydrodynamic virial coefficient $\left(k_{\mathrm{D}}\right)$ estimated from the initial slopes in Figure 5 as a function of $C_{\mathrm{SDS}} / C_{\mathrm{PEO}}$. Data in $0.1 \mathrm{M} \mathrm{NaCl}$. (b, bottom) Apparent second virial coefficients $\left(A_{2}\right)$ obtained from intensity light scattering (see text) as a function of $C_{\mathrm{SDS}} / C_{\mathrm{PEO}}$.

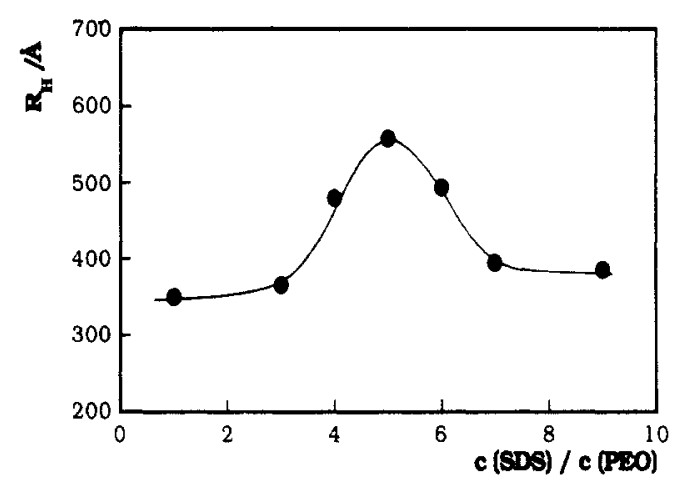

Figure 7. Hydrodynamic radius as a function of $C_{\mathrm{SDS}} / C_{\mathrm{PEO}} ; 0.1$ $\mathrm{M} \mathrm{NaCl}$ and $25^{\circ} \mathrm{C}$.

well with the value obtained for SDS alone in aqueous solution at that ionic strength; see below in Figure 13b.

Figure 10 depicts results for two relative concentrations: $C_{\mathrm{SDS}} / C_{\mathrm{PEO}}=3$, which is probably close to saturation at $0.01 \mathrm{M} \mathrm{NaCl}$ and for $C_{\mathrm{SDS}} / C_{\mathrm{PEO}}=9$ at $0.01 \mathrm{M} \mathrm{NaCl}$. At $C_{\mathrm{SDS}} / C_{\mathrm{PEO}}=3$, only a very small intensity contribution from SDS micelles can be observed, while at $C_{\mathrm{SDS}} / C_{\mathrm{PEO}}$ $=9$ the concentration of free SDS micelles is substantial. Due to the very different scattering intensities of the SDS micelles and the complex in the present system, the relaxation time distributions can at best only yield a very approximate estimate of the relative concentrations of the components.

An interesting comparison is provided by Figure 11; when the salt concentration is increased from 0.01 to 0.1 $\mathrm{M}$, the degree of SDS binding to PEO at $C_{\mathrm{PEO}}=0.1 \%$ is greatly increased as indicated by the strong decrease in the peak area of the SDS peak. This is the expected result
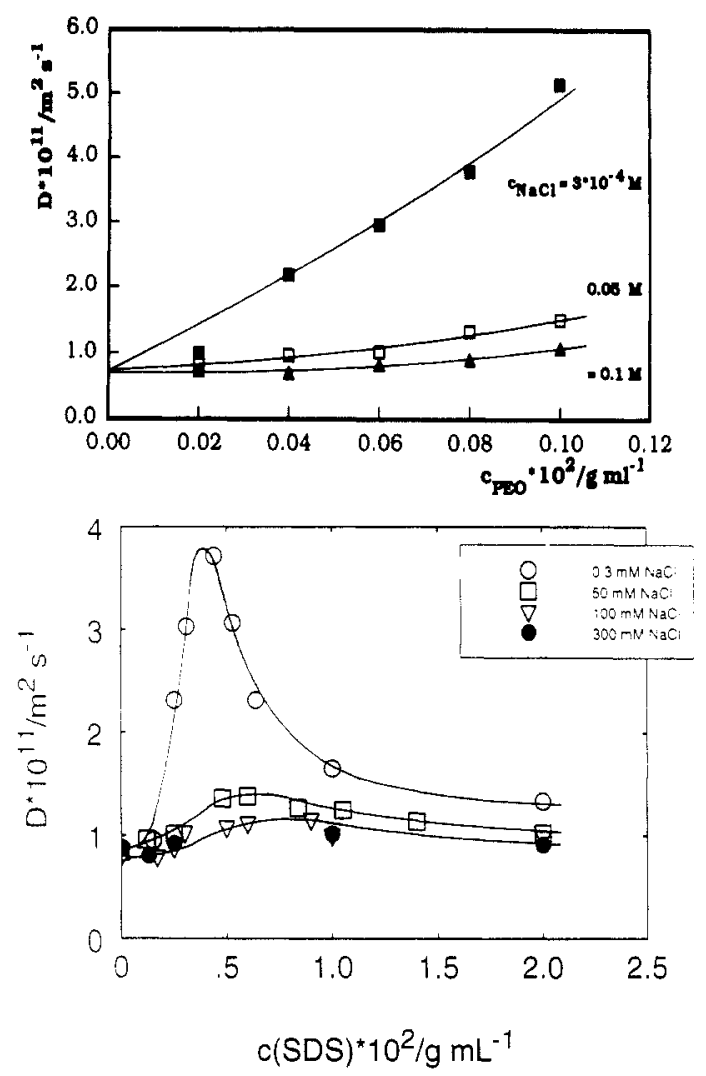

Figure 8. (a, top) Diffusion coefficient as a function of $C_{P B O}$ at $C_{\mathrm{SDS}} / C_{\mathrm{PEO}}=5$ at different ionic strengths. Date were obtained at $25^{\circ} \mathrm{C}$ and angle $60^{\circ}$. (b, bottom) Diffusion coefficients for the PEO-SDS complex as a function of $C_{\mathrm{SDS}}$ at a constant concentration $C_{\mathrm{PEO}}=0.1 \%$. Data were obtained at different ionic strengths, $25^{\circ} \mathrm{C}$, and at angle $60^{\circ}$.

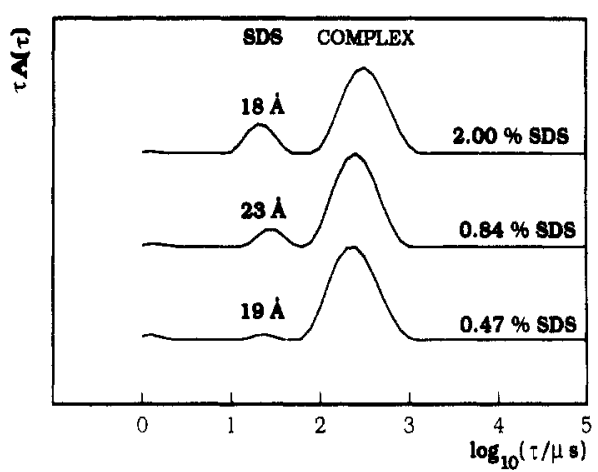

Figure 9. Relaxation time distributions obtained by ILT for the SDS/PEO complex as a function of SDS concentration at ionic strength $0.05 \mathrm{M}$. Constant $\mathrm{PEO}$ concentration $C_{\mathrm{PEO}}=0.1 \%$ and $25^{\circ} \mathrm{C}$.

since it is known that the aggregation numbers of both the PEO-bound micelles and micelles in polymer-free systems increase with the salt concentration; ${ }^{11,12}$ however, free micelles grow in size more than the bound micelles.

Francois et al. ${ }^{13}$ have also reported aggregation numbers for the bound SDS micelles. In $0.1 \mathrm{M} \mathrm{NaCl}$ a value of approximately 60 was reported over a range of SDS concentrations $(0.2-0.6 \%)$.

It is noted that free SDS micelles are still observed even at low $C_{\text {SDS. }}$ The cmc for SDS is approximately $0.043 \%$ $(1.5 \mathrm{mM})$ in $0.1 \mathrm{M} \mathrm{NaCl}$ in the absence of $\mathrm{PEO}$ and $0.038 \%$ in the presence of $0.1 \% \mathrm{PEO}$ at the same ionic strength as shown in Figure 12 for surface tension data for the SDS/PEO system. The cmc for SDS is reduced strongly as the $\mathrm{NaCl}$ concentration is increased. The cmc-lowering effect of the polymer is sensitive to the nature of the cation as shown by Dubin et al. ${ }^{15}$ 

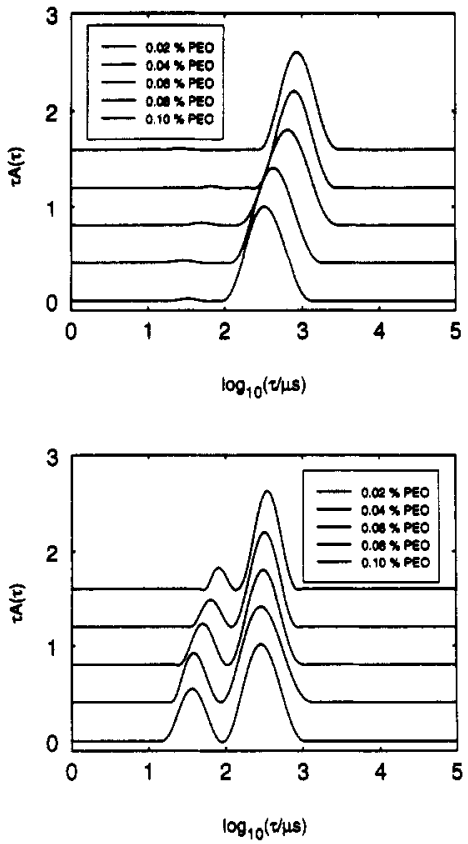

Figure 10. Relaxation time distributions using ILT for different $\mathrm{PEO}$ concentrations at $0.01 \mathrm{M} \mathrm{NaCl}$ and $25^{\circ} \mathrm{C}$ : (a, top) at $C_{\text {SDS }}$ / $C_{\mathrm{PEO}}=3$; (b, bottom) $C_{\mathrm{SDS}} / C_{\mathrm{PEO}}=9$.
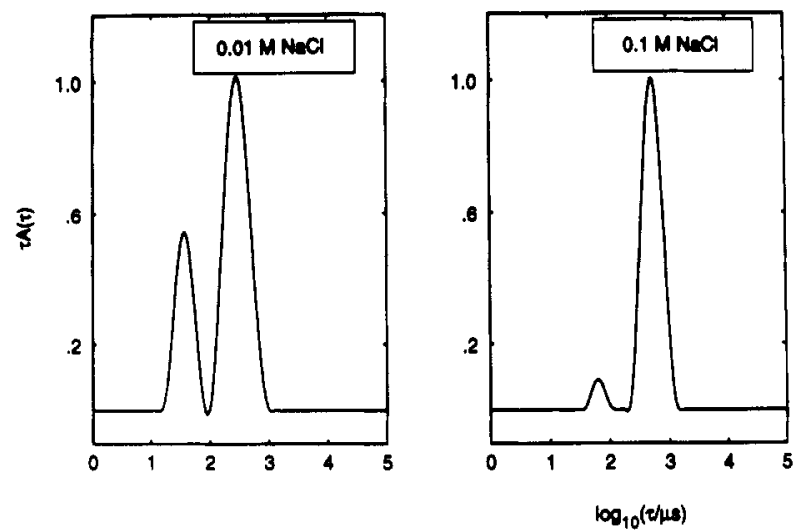

Figure 11. Influence of $\mathrm{NaCl}$ concentration: relaxation time distributions at $C_{\mathrm{SDS}} / C_{\mathrm{PEO}}=9$ with $C_{\mathrm{PEO}}=0.1 \%$. Data were obtained at $25^{\circ} \mathrm{C}$.

Aqueous SDS Solutions. Complementary measurements were made on SDS in $\mathrm{NaCl}$ solution. Figure 13a shows $D$ as a function of SDS concentration at three salt concentrations. The apparent hydrodynamic radius increases as the salt concentration is raised from a value of $16 \AA$ in $0.3 \mathrm{mM} \mathrm{NaCl}$ to $27 \AA$ in $100 \mathrm{mM} \mathrm{NaCl}$ although, as shown by fluorescence probing, this increase is not due primarily to a greater aggregation number, but probably depends on changes in hydration. These data are in good agreement with those of Corti and Degiorgio. ${ }^{28}$ Typical relaxation time distributions are depicted in Figure 13b for data at different SDS concentrations in the range up to $5 \%$ in $0.01 \mathrm{M} \mathrm{NaCl}$. The distributions were always single-peaked and no secondary aggregates are present.

\section{Conclusions}

Static and dynamic light scattering results are used here to illustrate the pronounced polyelectrolyte effect which is imparted to the neutral PEO chain on formation of a charged complex with the ionic surfactant SDS. The strong chain expansion, illustrated by changes in the radius of gyration and the relative viscosity, is reduced in the system when the saturation point at about SDS/PEO = 5 is exceeded and free counterions from the excess SDS are present.
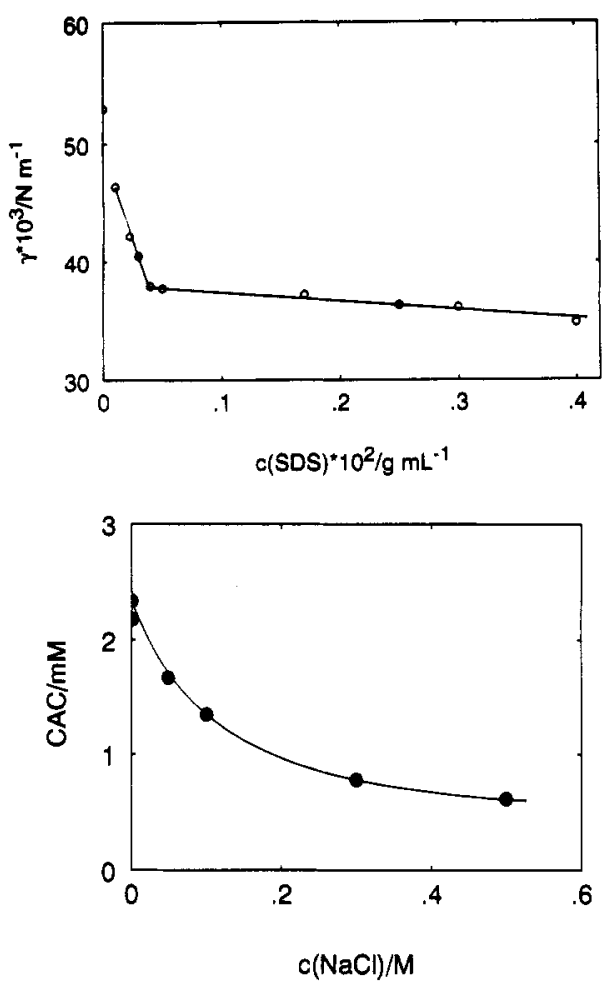

Figure 12. (a, top) Determination of critical aggregation concentration (cac) for the PEO/SDS system in $0.1 \mathrm{M} \mathrm{NaCl}$. cac $=0.038 \%$ ( $\mathrm{cmc}=0.043 \%$ in the absence of PEO). (b, bottom) Dependence of the cac on concentration $\mathrm{NaCl}$.
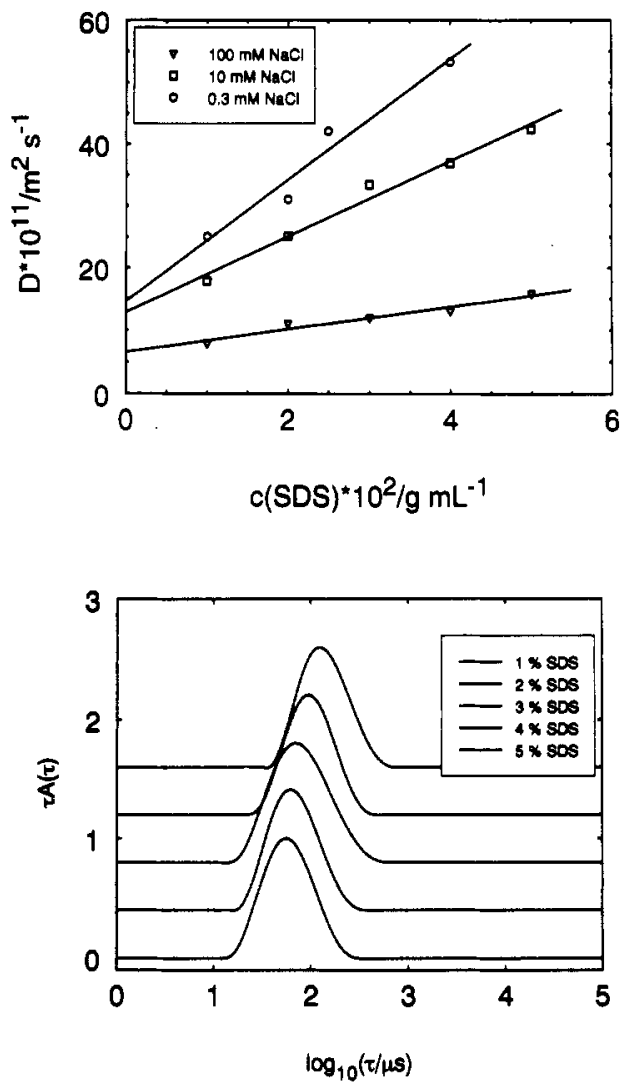

Figure 13. (a, top) Concentration dependence of diffusion coefficients for SDS micelles in media of different ionic strength at $25^{\circ} \mathrm{C}$. (b, bottom) Relaxation time distributions using ILT for SDS micelles at different concentrations in $0.01 \mathrm{M} \mathrm{NaCl}$ at $15^{\circ} \mathrm{C}$. Concentrations from top to bottom are in the sequence shown.

The diffusion coefficients also pass through a strong maximum owing to the charge fluctuations in the macroion solution. 
Relaxation time distributions obtained through Laplace inversion of the autocorrelation curves clearly show wellseparated contributions from the charged complex and free SDS micelles. These data reveal the sensitivity of the binding level to the ionic strength of the medium.

Acknowledgment. This work was supported by Carl Tryggers Fond, Stockholm, Sweden, and the Swedish National Board for Technical Development (NUTEK). Mats Almgren and Jan van Stam are thanked for discussions during the course of this work.

\section{References and Notes}

(1) Goddard, E. D. Colloids Surf. 1986, 19, 25.

(2) Saito, S. In Nonionic Surfactants; Schick, M. J., Ed.; Marcel Dekker, Inc.: New York, 1987; p 881.

(3) Hayakawa, K.; Kwak, J.C.T. In Cationic Surfactants; Rubingh, D. N., Holland, P. M., Eds.; Marcel Dekker, Inc.: New York, 1991; p 189

(4) Lindman, B.; Thalberg, K. In Polymer-Surfactant Interactions; Goddard, E. D., Ananthapadmanabhan, K. P., Eds.; CRC Press: Boca Raton, FL.

(5) Winnik, F. M.; Winnik, M. A. Polym. J. 1990, 22, 482.

(6) Winnik, F. M.; Winnik, M. A.; Tazuke, S. J.Phys. Chem. 1987, $91,594$.

(7) Carlsson, A.; Karlström, G.; Lindman, B. J. Phys. Chem. 1987, $93,3673$.

(8) Jones, M. N. J. Colloid Interface Sci. 1967, 23, 36.

(9) Chu, D., Thomas, J. K. J. Am. Chem. Soc. 1986, 108, 6270.

(10) (a) Cabane, B. J. Phys. Chem. 1977, 81, 1639. (b) Cabane, B. Duplessix, R. J. Phys. (Paris) 1982, 43, 1529. (c) Cabane, B.; Duplessix, R. J. Phys. (Paris) 1987, 48, 651.
(11) Zana, R.; Lianos, P.; Lang, J. J. Phys. Chem. 1985, 89, 41.

(12) van Stam, J.; Almgren, M.; Lindblad, C. Prog. Colloid Polym. Sci. 1991, 84, 13.

(13) Francois, J.; Dayantis, J.; Sabbadin, J. Eur. Polym. J. 1985, 21, 165.

(14) Shirahama, K. Colloid Polym. Sci. 1974, 252, 978.

(15) Dubin, P. L.; Gruber, J. H.; Jiulin, X.; Zhang, H. J. Colloid Interface Sci. 1992, 148, 35.

(16) Meewes, M.; Ricka, J.; de Silva, M.; Nyffenegger, R.; Binkert, Th. Macromolecules 1991, 24, 5811.

(17) Ricka, J.; Meewes, M.; Nyffenegger, R.; Binkert, Th. Phys. Rev. Lett. 1990, 65, 657.

(18) Dubin, P. L.; Rigsbee, D. R.; Gan, L.-M.; Fallon, M. A. Macromolecules 1988, 21, 2555.

(19) Brown, W.; Schillén, K.; Almgren, M.; Hvidt, S.; Bahadur, P. J. Phys. Chem. 1991, 95, 1850.

(20) Jakes, J. Czech. J. Phys. 1988, B38, 1305.

(21) Provencher, S. W. Makromol. Chem. 1979, 180, 201.

(22) Johnsen, R. M.; Brown, W. In Laser Light Scattering in Biochemistry; Harding, S. E., Sattelle, D. B., Bloomfield, V. A., Eds.; Royal Society of Chemistry: London, 1992; p 77.

(23) Polik, W. F.; Burchard, W. Macromolecules 1983, 16, 978.

(24) Pike, E. R.; Pomeroy, W. R. M.; Vaughan, J. M. J. Chem. Phys. $1975,62,3188$

(25) Tornberg, E. J. Colloid Interface Sci. 1977, 60, 50.

(26) Dubin, P. L.; Thé, S.S.; Gan, L. M.; Chew, C. H. Macromolecules $1990,23,2500$.

(27) Felderhof, B. U. J. Phys. A: Math. Gen. 1978, 11, 929.

(28) Corti, M.; Degiorgio, V. J. Phys. Chem. 1981, 85, 711.

Registry No. PEO, 25322-68-3; SDS, 151-21-3; NaCl, 764714-5. 